Canad. Math. Bull. Vol. 22 (1), 1979

\title{
A CENTRAL LIMIT THEOREM FOR GENERAL STOCHASTIC PROCESSES
}

\author{
BY \\ DUDLEY PAUL JOHNSON
}

\begin{abstract}
We show that under mild conditions the Central Limit Theorem holds for general stochastic processes.
\end{abstract}

Let $M(\Omega, F)$ be the Banach space of all measures on the measurable space $(\Omega, F)$ of all functions $\omega$ mapping the nonnegative integers into a measurable space $(S, \Sigma)$ where $F$ is the $\sigma$-field generated by the events $X_{n}(\omega)=\omega(n) \in U \in$ $\Sigma$. Let $T$ be the linear operator on $M(\Omega, F)$ defined by

$$
T \varphi\left(X_{1} \in U_{1}, \ldots, X_{n} \in U_{n}\right)=\varphi\left(X_{2} \in U_{1}, \ldots, X_{n+1} \in U_{n}\right),
$$

let $E(U), U \in \Sigma$ be the resolution of the identity

$$
E(U) \varphi(\Lambda)=\varphi\left(X_{0} \in U, \Lambda\right),
$$

and let $p^{*}$ be the linear functional

$$
p^{*} \varphi=\varphi(\Omega) .
$$

Suppose that $\Phi$ is a linear subspace of $M(\Omega, F)$ which is closed under the operators $T, E(U), U \in \Sigma$; that $\Phi^{*}$ is the Banach space of all bounded linear functionals on $\Phi$; and that $T^{*}$ and $E(U)^{*}, U \in \Sigma$ are the adjoints of the operators $T$ and $E(U), U \in \Sigma$. Then, noting that the spectrum of $T^{*}$ contains 1 , that $p^{*}$ is an eigenfunction corresponding to 1 of $T^{*}$ and that $\int e^{i u x} T^{*} E(d x)^{*}$ converges to $T^{*}$ as $u \rightarrow 0$, we have the following Central Limit Theorem.

THEOREM. If the operator $\int e^{i u x} T^{*} E(d x)^{*}$ has an eigenvalue of the form $1+i a u+b u^{2}+o\left(u^{2}\right)$ with a corresponding eigenfunction $e_{u}^{*}$ which converges to $p^{*}$ as $u \rightarrow 0$ in the norm topology of $\Phi^{*}$, then for all $\varphi \in \Phi$ with $p^{*} \varphi=1$ we have

$$
\lim _{n \rightarrow \infty} \varphi\left[\frac{X_{1}+\cdots+X_{n}-n a}{\sqrt{n}\left(a^{2}+2 b\right)}<x\right]=\frac{1}{\sqrt{2 \pi}} \int_{-\infty}^{x} e^{-\left(u^{2} / 2\right)} d u
$$

Proof. Noting that for each $\varphi \in \Phi$

$$
\varphi\left[X_{1} \in U_{1}, \ldots, X_{n} \in U_{n}\right]=p^{*} E\left(U_{n}\right) T \cdots E\left(U_{1}\right) T \varphi,
$$

Received by the editors November 28, 1977 and, in revised form, May 31, 1978. 
we have

$$
\begin{aligned}
& \lim _{n \rightarrow \infty} \int \exp \left(i u \frac{X_{1}+\cdots+X_{n}-n a}{\sqrt{n}\left(a^{2}+2 b\right)}\right) d \varphi \\
& =\lim _{n \rightarrow \infty} \int \ldots \int \exp \left(i u \frac{x_{1}+\cdots+x_{n}-n a}{\sqrt{ } n\left(a^{2}+2 b\right)}\right) p^{*} E\left(d x_{n}\right) T \cdots E\left(d x_{1}\right) T \varphi \\
& =\lim _{n \rightarrow \infty}\left[\int \exp \left(i u \frac{x-a}{\sqrt{n\left(a^{2}+2 b\right)}}\right) T^{*} E(d x)^{*}\right]^{n} p^{*} \varphi \\
& =\lim _{n \rightarrow \infty}\left[\exp \left(-i u \frac{a}{\sqrt{n\left(a^{2}+2 b\right.}}\right)\right]^{n}\left[\int \exp \left(i u \frac{x}{\sqrt{n\left(a^{2}+2 b\right)}}\right) T^{*} E(d x)^{*}\right]^{n} \\
& \times e^{*} \frac{u}{\sqrt{n}\left(a^{2}+2 b\right)} \varphi \\
& =\lim _{n \rightarrow \infty}\left[1-i u \frac{a}{\sqrt{ } n\left(a^{2}+2 b\right)}-\frac{u^{2} a^{2}}{2 n\left(a^{2}+2 b\right)}+o\left(\frac{1}{n}\right)\right]^{n} \\
& \times\left[1+i a \frac{u}{\sqrt{n\left(a^{2}+2 b\right)}}+b \frac{u^{2}}{n\left(a^{2}+2 b\right)}+o\left(\frac{1}{n}\right)\right]^{n} e^{*} \frac{u}{\sqrt{n}\left(a^{2}+2 b\right)} \varphi \\
& =\lim _{n \rightarrow \infty}\left[1-\frac{u^{2}}{2 n}+o\left(\frac{1}{n}\right)\right]^{n} e_{u / \sqrt{n}\left(a^{2}+2 b\right)}^{*} \varphi \\
& =e^{-\left(u^{2} / 2\right)} p^{*} \varphi
\end{aligned}
$$

and the theorem is proved.

\section{REFERENCES}

1. Dunford, N. and Schwartz, S. (1958). Linear Operators I: General theory. Pure and Appl. Math., Vol. 7, Interscience, New York.

2. Johnson, Dudley Paul, (1970). Markov Process representations of general stochastic processes. Proc. Amer. Math. Soc. 24, 735-738.

3. Johnson, Dudley Paul, (1974). Representations and classifications of stochastic processes. Trans. Amer. Math. Soc. 188 (1974), 179-197.

4. Meyer, P. A. (1966). Probability and potentials. Blaisdell, Waltham, Mass.

Department of Mathematics and Statistics

ThE University OF CALGARY

Calgary, Alberta T2N 1N4 